\title{
Single scan TROSY and E.COSY suite of experiments for the measurement of residual dipolar couplings in proteins
}

\author{
Tommaso Eliseo ${ }^{\mathrm{a}}$, Mariana Gallo ${ }^{\mathrm{a}}$, Riccardo Melis ${ }^{\mathrm{a}}$, Maurizio Paci ${ }^{\mathrm{a}}, \mathrm{Renzo}_{\mathrm{Bazzo}}{ }^{\mathrm{b}}$ and \\ Daniel O. Cicero ${ }^{\mathrm{a}, *}$ \\ a Department of Chemical Science and Technology, University of Rome "Tor Vergata", \\ via della Ricerca Scientifica 1, 00133 Rome, Italy \\ ${ }^{\mathrm{b}}$ IRBM “P. Angeletti”, Via Pontina km 30.600, 00040, Pomezia, Rome, Italy
}

\begin{abstract}
Simple modifications of the gradient enhanced version of the TROSY experiment allow to obtain four different spectra containing each one of the components of the $\mathrm{H}-\mathrm{N}$ doublet in ${ }^{15} \mathrm{~N}$ labelled proteins. By measuring the difference in peak position among these four spectra, residual dipolar coupling values can be obtained for medium sized proteins. This experiment, which exploits the use of pulse field gradients to select the ${ }^{15} \mathrm{~N}$ coherence pathway, produces excellent results in terms of water suppression. Moreover, tuning of a single delay in the sequence reduces notably the presence of artifacts. The performance of this suite of experiments, that we called TEC (Trosy-E.Cosy) experiment, is tested against a $J$-modulation method, inherently more accurate but more time consuming, for the accuracy in the observed values of residual dipolar couplings. For larger proteins, the use of this strategy allows to select the most favourable combination of peaks giving the sharpest signals.
\end{abstract}

Keywords: NMR, residual dipolar couplings, TROSY, eCOSY

\section{Introduction}

Residual dipolar couplings (RDCs) constitute a valuable piece of information in the process of studying macromolecular structure, dynamics and interaction by NMR (for a recent review see [1]). They contain orientational information of internuclear vectors with respect to the external magnetic field. Their use in conjunction with more classical constraints, like interproton distances and dihedral angles, is changing the way structural studies by NMR are conducted. They can be measured in samples that are partially oriented with respect to the magnetic field by means of different media [2] or by the use of paramagnetic tags [3]. Nowadays, different types of RDCs are measurable, including $\mathrm{HN}-\mathrm{N}, \mathrm{H} \alpha-$ $\mathrm{C} \alpha, \mathrm{C} \alpha-\mathrm{C} \beta, \mathrm{C} \alpha-\mathrm{C}^{\prime}, \mathrm{N}-\mathrm{C}^{\prime}, \mathrm{HN}-\mathrm{C}^{\prime}, \mathrm{H}-\mathrm{H}$ among others [4]. A prominent role is played by the $\mathrm{HN}-\mathrm{N}$ coupling constants for a number of reasons: they are easily measured using ${ }^{15} \mathrm{~N}$-only labelled proteins, resolution of $\mathrm{HN}$ peaks is very favourable due to the spread in chemical shift of the ${ }^{15} \mathrm{~N}$ nuclei, their value is relatively large also in case of modest alignment (is only second to the $\mathrm{H} \alpha-\mathrm{C} \alpha \mathrm{RDC}$ ), and,

\footnotetext{
${ }^{*}$ Corresponding author: Daniel O. Cicero, Department of Chemical Science and Technology, University of Rome "Tor Vergata", via della Ricerca Scientifica 1, 00133 Rome, Italy. Tel.: +39 067259 4835; Fax: +39 067259 4328; E-mail: cicero@ scienze.uniroma2.it.
} 
being the ${ }^{15} \mathrm{~N} T_{2}$ longer compared to other nuclei in the protein, their use can be extended also to large proteins [5].

As with most of the other types of RDCs, the available methods for the measurement of $\mathrm{HN}-\mathrm{N}$ dipolar couplings can be classified according to whether the coupling is observed as a difference in line frequencies or in the manner of a quantitative $J$ experiment [6,7], where the coupling is encoded in the signal intensity. Regarding the first type, the determination of single-bond $J$ splittings from the separation of doublets is useful for well resolved spectra of small proteins. However, due to the doubling of resonance lines, it becomes more difficult to resolve peaks as protein size increases. This problem can be alleviated somewhat by collecting spectra in which each individual doublet component is recorded separately. In the simplest approach, two spectra are added or subtracted to achieve the spin-state selection. In fact, the two doublet components can be described as the sum or difference of in-phase and antiphase magnetization, $I_{y} \pm 2 I_{y} S_{z}$. This is the base of the IPAP (In Phase-Anti Phase) method [8], which is one of the most used approaches to separate the two components. A number of other filters have been described [9]. In the DIPSAP (Double In Phase-Single Anti Phase) approach [10], two in-phase (with opposite sign) and one anti-phase spectra are obtained. Two different linear combinations are then obtained, yielding the two subspectra. The result is less sensitive to $J$-mismatches, that are likely to occur specially in the aligned sample, where very different $\mathrm{H}-\mathrm{N}$ coupling constants are observed.

One special case of spin-state selection scheme is the TROSY experiment [11]. In fact, the original sequence was devised to select only the slowly relaxing component, which is one of the four components of the H-N spin system. In its original form the TROSY sequence used a 8-step phase cycling scheme to eliminate the three non desired components and to produce the quadrature detection in the ${ }^{15} \mathrm{~N}$ dimension. Subsequent introduction of the "Rance-Kay" sensitivity-enhancement feature [12] allowed the phase-cycling to be reduced to 4 steps [13]. In addition, two versions of a single-scan TROSY experiments, using gradient selection and sensitivity enhancement were presented [14,15]. The fact that the TROSY experiment is particularly well suited to perform a spin state filter, opened the possibility of its use to measure RDCs. Sensitivity-enhanced versions of the TROSY-anti-TROSY, and E.COSY experiments for measuring one-bond ${ }^{1} \mathrm{H}-{ }^{15} \mathrm{~N}$ couplings were presented [16]. In these experiments, coherence gradient selection was avoided in order to obtain in a single spectrum both the TROSY and the anti-TROSY peaks, or the two components of the E.COSY system. The two subspectra were separated by the insertion of a TPPI scheme, which confers an artificial ${ }^{15} \mathrm{~N}$ resonance offset.

We have combined the best of the two approaches in a single suite of experiments, that we called TEC (Trosy-E.Cosy) experiment. The use of gradient selection in combination with suitable pulse phases, selects one of the four components in the single scan. It is sufficient to invert a gradient polarity and/or ${ }^{a}{ }^{1} \mathrm{H}$ pulse phase to allow the selection of any of the four components. The sequence, which makes use of coherence selection by gradients, presents also the extra advantage of producing a better water suppression. Elimination of artifacts present in the TROSY experiments will be also discussed. The measurement of RDCs using this suite of experiments will be exemplified on two different samples. The first is constituted by a medium size protein $(15 \mathrm{kDa})$, chenodeoxycholate complexed with the chicken liver bile acid binding protein [17], at relatively low field (400 MHz), and the second by a larger protein (30 kDa), Azotobacter vinelandii Rhodanese [18], at higher field (700 MHz). These two examples will be used to illustrate the versatility inherent to the method that allows to use those peaks that are best suited for the coupling constant measurement, depending on the magnitude of the TROSY effect. 


\section{Experimental procedure}

All experiments were conducted at $400.13 \mathrm{MHz}$ or $700.09{ }^{1} \mathrm{H}$ frequency on Bruker Avance spectrometers equipped with a triple band probe and $z$-gradient accessory, at $298 \mathrm{~K}$. Data were processed using the NMRPipe software [19], and visualized and analyzed using NMRView [20].

\section{1. cl-BABP experiments}

Data were acquired at $400 \mathrm{MHz}$. Isotropic data were collected from a $1.1 \mathrm{mM}$ uniformly ${ }^{15} \mathrm{~N},{ }^{13} \mathrm{C}$ labeled sample of chicken liver Bile Acid Binding Protein (cl-BABP) complexed with unlabeled chenodeoxycholate, in $95 \% \mathrm{H}_{2} \mathrm{O}, 5 \% \mathrm{D}_{2} \mathrm{O}$ containing $30 \mathrm{mM}$ sodium phosphate buffer, $\mathrm{pH} 7.0$, and DTT $5 \mathrm{mM}$. Anistropic data were collected dissolving the protein in $14 \mathrm{mg} / \mathrm{ml}$ liquid crystalline Pf1 (Asla Labs) as alignment medium [21]. It was necessary to add sodium chloride up to $150 \mathrm{mM}$ in order to decrease the signal linewidth. In the TEC experiment, 256 complex points in $F_{1}$ and 2048 complex points in $F_{2}$ were acquired for each subspectra. The spectral width was $1419.235 \mathrm{~Hz}$ and $6410.256 \mathrm{~Hz}$ in $F_{1}$ and in $F_{2}$, respectively. The carrier frequency was placed on water resonance (4.688 ppm) in $F_{2}$. Experiments were collected in echo-antiecho combination to obtain sensitivity enhancement and were processed as described [22]. Identical spectral processing was applied to all TROSY-type experiments. Gaussian apodization was employed in both dimensions prior to zero filling. $F_{2}$ dimension was zero filled to $4 k$ points and $F_{1}$ dimension was zero filled to 512 points. Values of ${ }^{15} \mathrm{~N}-{ }^{1} \mathrm{H}$ coupling constants were extracted measuring the difference in frequency position of corresponding peaks in the TROSY and antiTROSY spectra along both dimensions. The same measure was performed in the case of the two E.COSY spectra. RDCs were obtained by subtraction of coupling constants measured in the isotropic phase from the corresponding constants measured in the aligned sample.

$J$-modulated spectra were collected in interleaved fashion using the same spectral widths of TROSYtype experiments and using a published pulse sequence [23]. A total of 132 complex points in $F_{1}$ and 2048 complex points in $F_{2}$ were collected. Quadrature detection in $F_{1}$ was achieved with States-TPPI method. The total $J$-coupling evolution time was $17.6 \mathrm{~ms}$ using 15 sampling points equally separated $(1.1 \mathrm{~ms})$. Peak intensities were measured and plotted to fit the appropriate equations as described in the original paper [23].

\subsection{Rhodanese experiments}

NMR data were collected from a $1.0 \mathrm{mM}$ uniformly ${ }^{2} \mathrm{H},{ }^{15} \mathrm{~N}$, and ${ }^{13} \mathrm{C}$-labeled sample of Rhda $95 \%$ $\mathrm{H}_{2} \mathrm{O}, 5 \% \mathrm{D}_{2} \mathrm{O}$ containing $100 \mathrm{mM}$ d-Tris- $\mathrm{HCl}(\mathrm{pH} 7.20), 0.3 \mathrm{M} \mathrm{NaCl}, 10 \mathrm{mM}$ thiosulfate buffer and $0.2 \%$ deuterated octil- $\beta$-D-glucoside at $25^{\circ} \mathrm{C}$. NMR spectra were recorded at $700 \mathrm{MHz}$. The IPAP experiment was implemented as described in the original work [8]. Both for the IPAP and TEC experiments, a total of 1024 by 150 complexes were obtained. Spectral width were set to 2837.9 and $11261.2 \mathrm{~Hz}$ in $F_{1}$ and $F_{2}$ dimensions, respectively. The total measurement time for each of the two experiments were $8 \mathrm{~h} 40 \mathrm{~min}$. Data processing was performed as already described for cl-BABP spectra.

\section{Results and discussion}

\subsection{Pulse sequence description}

Figure 1a shows the sequence used to obtain each of the four components of the HN-N system in four different spectra. It is basically the sequence of the gradient-selected sensitivity enhanced TROSY 


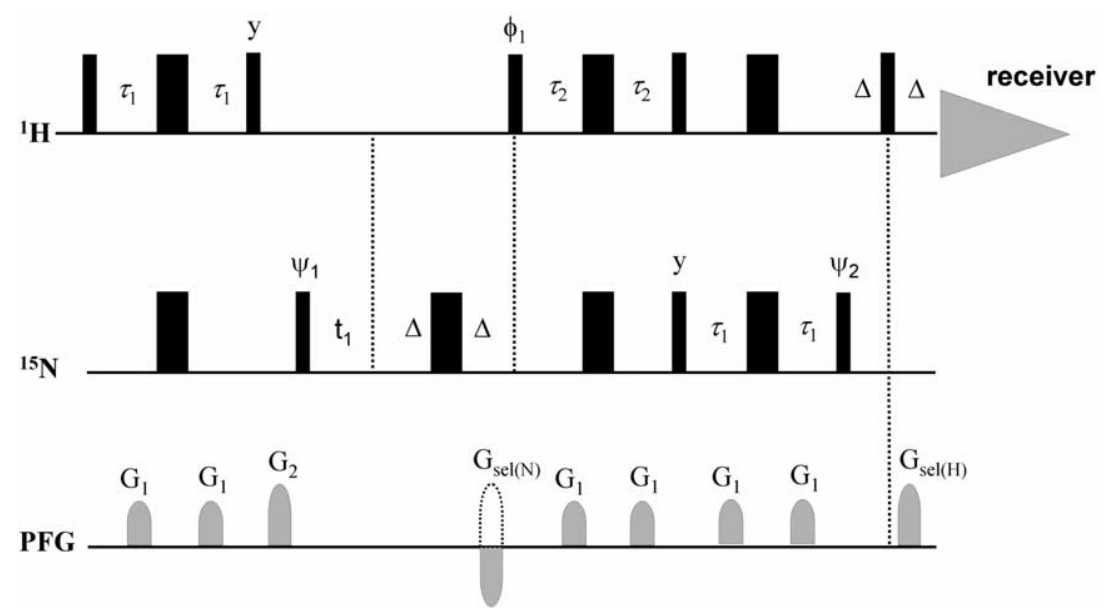

(a)

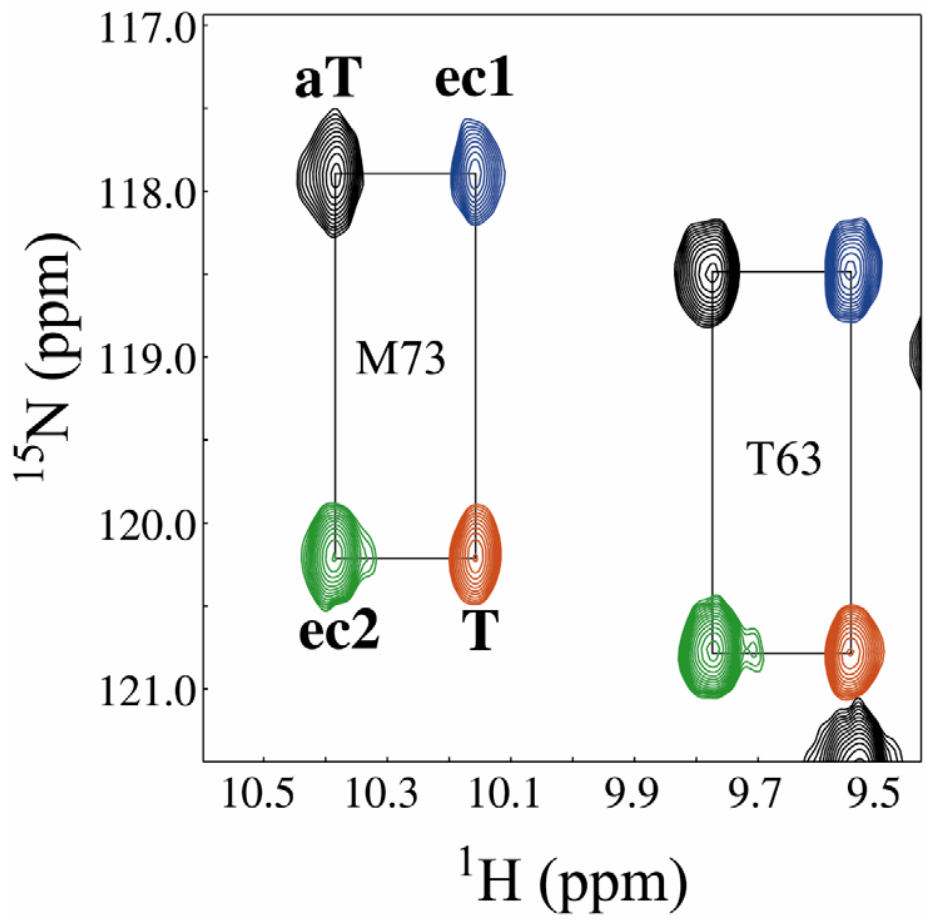

(b)

Fig. 1. (a) Experimental scheme for single scan, sensitivity-enhanced TEC two-dimensional ${ }^{1} \mathrm{H},{ }^{15} \mathrm{~N}$ correlation spectroscopy. Open and filled rectangles represent $90^{\circ}$ and $180^{\circ}$ hard rf-pulses, respectively. All pulses are applied in the $x$ direction if not stated otherwise. $\tau_{1}$ is set to $1 / 4^{1} J_{\mathrm{HN}}=2.68 \mathrm{~ms}, \tau_{2}=2.20 \mathrm{~ms}$ (see text). Pulse field gradients are applied along the $\mathrm{z}$ axis using a sine bell-shaped envelope with following amplitude and duration: $G_{1}, 15 \mathrm{G} / \mathrm{cm}, 1.6 \mathrm{~ms} ; G_{2}, 40 \mathrm{G} / \mathrm{cm}, 1.6 \mathrm{~ms}$; $G_{3}, 22.5 \mathrm{G} / \mathrm{cm}, 1.6 \mathrm{~ms} ; G_{4}, 25 \mathrm{G} / \mathrm{cm}, 1.6 \mathrm{~ms} ; G_{\mathrm{N}}, 40 \mathrm{G} / \mathrm{cm}, 1 \mathrm{~ms} ; G_{\mathrm{H}}, 0.202 \mathrm{G} / \mathrm{cm}, 1 \mathrm{~ms}$. The gradients $G_{\mathrm{N}}$ and $G_{\mathrm{H}}$ are placed in spin-echo blocks to avoid chemical shift evolution. The use of gradients $G_{\mathrm{N}}$ and $G_{\mathrm{H}}$ allows the recording of pure phase absorption spectrum without any cycling of the pulse phases. Frequency discrimination in $F_{1}$ is achieved by using the gradient-enhanced sensitivity method in which two separate data sets are recorded for each $t_{1}$ increment with 180 degree added to $\psi_{1}$ and the sign of $G_{\mathrm{N}}$ gradient inverted for the second set. As described in the text, appropriate combination of the encoding $G_{\mathrm{N}}$ gradient polarity and the ${ }^{1} \mathrm{H}$ pulse $\phi_{1}$ phase gives rise to only one of the four $J$-coupled components of the 
experiment [15]. The product operator formalism [24] can be used to explain how, by changing the polarity of the encoding gradient $G_{\text {selN }}$ and the phase $\phi_{1}$ of a $90^{\circ}{ }^{1} \mathrm{H}$ pulse, it is possible to select one of the four corresponding magnetization terms, whose nomenclature is defined in Fig. 1b. A convenient way to handle single transition components is to rewrite the magnetization term that is created in the first scan just before the $t_{1}$ evolution in the following manner:

$$
2 \mathrm{H}_{z} \mathrm{~N}_{x}=2 \mathrm{H}_{z} \mathrm{~N}_{x}+\mathrm{N}_{x} / 2-\mathrm{N}_{x} / 2=\left(\mathrm{N}_{x}+2 \mathrm{H}_{z} \mathrm{~N}_{x}\right) / 2-\left(\mathrm{N}_{x}-2 \mathrm{H}_{z} \mathrm{~N}_{x}\right) / 2 .
$$

In this way, the two terms describe the magnetization that evolves during $t_{1}$ with a phase angle of $\left(\Omega_{\mathrm{N}}+\right.$ $\left.\pi J_{\mathrm{HN}}\right)$ and $\left(\Omega_{\mathrm{N}}-\pi J_{\mathrm{HN}}\right)$, respectively, representing the high-frequency and low-frequency component of the doublet. After $t_{1}$ evolution, the different terms originating from (1) can be regrouped into:

$$
\begin{aligned}
& {\left[\left(\mathrm{N}_{x}+2 \mathrm{H}_{z} \mathrm{~N}_{x}\right) \cos \left(\omega_{\mathrm{N}} t_{1}+\pi J t_{1}\right)+\left(\mathrm{N}_{y}+2 \mathrm{H}_{z} \mathrm{~N}_{y}\right) \sin \left(\omega_{\mathrm{N}} t_{1}+\pi J t_{1}\right)\right] / 2} \\
& -\left[\left(\mathrm{N}_{x}-2 \mathrm{H}_{z} \mathrm{~N}_{x}\right) \cos \left(\omega_{\mathrm{N}} t_{1}-\pi J t_{1}\right)+\left(\mathrm{N}_{y}-2 \mathrm{H}_{z} \mathrm{~N}_{y}\right) \sin \left(\omega_{\mathrm{N}} t_{1}-\pi J t_{1}\right)\right] / 2 .
\end{aligned}
$$

The first term describes the real (phase $x$ ) and imaginary (phase $y$ ) parts of the high-frequency component of the doublet, and the second term represents the same two components for the low-frequency component. After gradient encoding with $G_{\text {selN }}$, the four magnetization components end up with the following coefficients:

$$
\begin{aligned}
& {\left[\left(\mathrm{N}_{x}+2 \mathrm{H}_{z} \mathrm{~N}_{x}\right)\left(\frac{1}{2}\left(G_{1}+G_{-1}\right) c^{+}-\frac{1}{2 i}\left(G_{1}-G_{-1}\right) s^{+}\right)\right.} \\
& \left.-\left(\mathrm{N}_{y}+2 \mathrm{H}_{z} \mathrm{~N}_{y}\right)\left(\frac{1}{2 i}\left(G_{1}-G_{-1}\right) c^{+}+\frac{1}{2}\left(G_{1}+G_{-1}\right) s^{+}\right)\right] / 2 \\
& -\left[\left(\mathrm{N}_{x}-2 \mathrm{H}_{z} \mathrm{~N}_{x}\right)\left(\frac{1}{2}\left(G_{1}+G_{-1}\right) c^{-}-\frac{1}{2 i}\left(G_{1}-G_{-1}\right) s^{-}\right)\right. \\
& \left.\quad-\left(\mathrm{N}_{y}-2 \mathrm{H}_{z} \mathrm{~N}_{y}\right)\left(\frac{1}{2 i}\left(G_{1}-G_{-1}\right) c^{-}+\frac{1}{2}\left(G_{1}+G_{-1}\right) s^{-}\right)\right] / 2,
\end{aligned}
$$

where

$$
\begin{aligned}
& G_{1}=\exp \left(-i \gamma_{\mathrm{N}} G_{\text {selN }}\right) \\
& G_{-1}=\exp \left(i \gamma_{\mathrm{N}} G_{\mathrm{selN}}\right) ; \\
& c^{ \pm}=\cos \left(\omega_{\mathrm{N}} t_{1} \pm \pi J t_{1}\right) ; \\
& s^{ \pm}=\sin \left(\omega_{\mathrm{N}} t_{1} \pm \pi J t_{1}\right)
\end{aligned}
$$

(continued) ${ }^{1} \mathrm{H}-{ }^{15} \mathrm{~N}$ multiplet. (b) Overlay of a selected region of the four single scan TROSY-type two-dimensional ${ }^{1} \mathrm{H},{ }^{15} \mathrm{~N}$ correlation spectra recorded on ${ }^{15} \mathrm{~N} /{ }^{13} \mathrm{C}$-labeled cl-BABP, which were obtained by combination of $G_{\mathrm{N}}$ gradient polarity and $\phi_{1}$ phase. For clarity cross-peaks originating from the four distinct spectra are displayed with different colours and are labeled accordingly: T (TROSY component, red), aT (antiTROSY, black), ec1 (lower ${ }^{1} \mathrm{H}$ frequency E.COSY component, blue), ec2 (upper ${ }^{1} \mathrm{H}$ frequency E.COSY component, green). $J$-couplings constants are measured as the difference in the peak frequency position in TROSY and anti TROSY spectra or, in the same way, as the difference in the peak frequency position in ec1 and ec2 spectra. 
Application of the whole set of pulses until the last decoding gradient $\left(G_{\mathrm{selH}}\right)$ and considering two different values for $\phi_{1}(y$ or $-y)$ will transform expression 3 into:

$$
\begin{aligned}
& {\left[\left(2 \mathrm{H}_{y} \mathrm{~N}_{z} \mp \mathrm{H}_{y}\right)\left(\frac{1}{2}\left(G_{1}+G_{-1}\right) c^{+}-\frac{1}{2 i}\left(G_{1}-G_{-1}\right) s^{+}\right)\right.} \\
& \left.-\left(-\mathrm{H}_{x} \pm 2 \mathrm{H}_{x} \mathrm{~N}_{z}\right)\left(\frac{1}{2 i}\left(G_{1}-G_{-1}\right) c^{+}+\frac{1}{2}\left(G_{1}+G_{-1}\right) s^{+}\right)\right] / 2 \\
& -\left[\left(2 \mathrm{H}_{y} \mathrm{~N}_{z} \pm \mathrm{H}_{y}\right)\left(\frac{1}{2}\left(G_{1}+G_{-1}\right) c^{-}-\frac{1}{2 i}\left(G_{1}-G_{-1}\right) s^{-}\right)\right. \\
& \left.\quad-\left(-\mathrm{H}_{x} \mp 2 \mathrm{H}_{x} \mathrm{~N}_{z}\right)\left(\frac{1}{2 i}\left(G_{1}-G_{-1}\right) c^{-}+\frac{1}{2}\left(G_{1}+G_{-1}\right) s^{-}\right)\right] / 2 .
\end{aligned}
$$

where the two signs refer to $\phi_{1}=y$ or $\phi_{1}=-y$, respectively. Expression (4) already shows that using $\phi_{1}=y$ exchanges the relative displacement from the center of the peak, showing the high-frequency component during $t_{1}$ (the first term of (4) which contains the $c^{+}$and $s^{+}$functions) and resonating at low-frequency during $t_{2}$ (because there is an opposite sign between the two terms that compose the magnetization), and vice versa. This is the characteristic of the trosy and anti-trosy peaks (peaks $\mathrm{T}$ and aT in Fig. 1b). On the other hand, use of $\phi_{1}=-y$ will keep the same relative displacement from the center during $t_{1}$ and $t_{2}$, which is a characteristic of the two e-cosy components (peaks ec1 and ec2, Fig. 1b). At this point, however, none of these terms are observable because they have an encoded phase represented by $G_{1}$ and $G_{-1}$ which depends on the position of the various nuclei along the $z$-direction. Application of the decoding gradient $G_{\text {selH }}$ gives rise to the following final expression, which represents the magnetization just before detection:

$$
\begin{aligned}
& \frac{1}{2}[(\left.\left(2 \mathrm{~N}_{z} \mathrm{H}_{x} \mp \mathrm{H}_{x}\right)\left(\frac{1}{2 i}\left(G_{2}-G_{-2}\right)\right)+\left(2 \mathrm{~N}_{z} \mathrm{H}_{y} \mp \mathrm{H}_{y}\right)\left(\frac{1}{2}\left(G_{2}+G_{-2}\right)\right)\right) \\
& \times\left(\left(\frac{1}{2}\left(G_{1}+G_{-1}\right)\right) c^{+}-\left(\frac{1}{2 i}\left(G_{1}-G_{-1}\right)\right) s^{+}\right) \\
&-\left(\left(-\mathrm{H}_{x} \pm 2 \mathrm{H}_{x} \mathrm{~N}_{z}\right)\left(\frac{1}{2}\left(G_{2}+G_{-2}\right)\right)+\left(\mathrm{H}_{y} \mp 2 \mathrm{H}_{y} \mathrm{~N}_{z}\right)\left(\frac{1}{2 i}\left(G_{2}-G_{-2}\right)\right)\right) \\
& \times\left.\left(\left(\frac{1}{2 i}\left(G_{1}-G_{-1}\right)\right) c^{+}+\left(\frac{1}{2}\left(G_{1}+G_{-1}\right)\right) s^{+}\right)\right] \\
&-\frac{1}{2}\left[\left(\left(2 \mathrm{~N}_{z} \mathrm{H}_{x} \pm \mathrm{H}_{x}\right)\left(\frac{1}{2 i}\left(G_{2}-G_{-2}\right)\right)+\left(2 \mathrm{~N}_{z} \mathrm{H}_{y} \pm \mathrm{H}_{y}\right)\left(\frac{1}{2}\left(G_{2}+G_{-2}\right)\right)\right)\right. \\
& \quad \times\left(\left(\frac{1}{2}\left(G_{1}+G_{-1}\right)\right) c^{-}-\left(\frac{1}{2 i}\left(G_{1}-G_{-1}\right)\right) s^{-}\right) \\
& \quad-\left(\left(-\mathrm{H}_{x} \mp 2 \mathrm{H}_{x} \mathrm{~N}_{z}\right)\left(\frac{1}{2}\left(G_{2}+G_{-2}\right)\right)+\left(\mathrm{H}_{y} \pm 2 \mathrm{H}_{y} \mathrm{~N}_{z}\right)\left(\frac{1}{2 i}\left(G_{2}-G_{-2}\right)\right)\right) \\
&\left.\quad \times\left(\left(\frac{1}{2 i}\left(G_{1}-G_{-1}\right)\right) c^{-}+\left(\frac{1}{2}\left(G_{1}+G_{-1}\right)\right) s^{-}\right)\right]
\end{aligned}
$$


where, by analogy with the definition of expression (3),

$$
\begin{aligned}
& G_{2}=\exp \left(-i \gamma_{\mathrm{H}} G_{\mathrm{selH}}\right) ; \\
& G_{-2}=\exp \left(i \gamma_{\mathrm{H}} G_{\mathrm{selH}}\right) .
\end{aligned}
$$

The two gradients $G_{\text {selN }}$ and $G_{\text {selH }}$ must be chosen in a way that some products of the functions $G_{1}, G_{-1}$ with $G_{2}$ and $G_{-2}$ become 1 . Only those terms will be transformed into observable magnetization. By choosing the following two conditions:

$$
\begin{aligned}
G_{\text {selN }}=-\frac{\gamma_{\mathrm{H}}}{\gamma_{\mathrm{N}}} G_{\text {selH }} & \text { condition (a), } \\
G_{\text {selN }}=\frac{\gamma_{\mathrm{H}}}{\gamma_{\mathrm{N}}} G_{\mathrm{selH}} & \text { condition (b), }
\end{aligned}
$$

that differ in the polarity of one of the two gradients, we will have the following unitary values for the cross products between the $G$ functions:

$$
\begin{array}{ll}
G_{1} G_{2}=G_{-1} G_{-2}=1 & \text { condition (a), } \\
G_{1} G_{-2}=G_{-1} G_{2}=1 & \text { condition (b). }
\end{array}
$$

Only terms of expression (5) that present the unitary $G$ cross products will be transformed into observable terms. We can calculate that the observable terms reduce to:

condition (a)

$$
\begin{aligned}
& \frac{1}{2}\left[\left(\left(2 \mathrm{~N}_{z} \mathrm{H}_{x} \mp \mathrm{H}_{x}\right) \frac{1}{2} s^{+}+\left(2 \mathrm{~N}_{z} \mathrm{H}_{y} \mp \mathrm{H}_{y}\right) \frac{1}{2} c^{+}\right)\right. \\
& \left.-\left(\left(-\mathrm{H}_{x} \pm 2 \mathrm{H}_{x} \mathrm{~N}_{z}\right) \frac{1}{2} s^{+}+\left(\mathrm{H}_{y} \mp 2 \mathrm{H}_{y} \mathrm{~N}_{z}\right)\left(-\frac{1}{2} c^{+}\right)\right)\right] \\
& -\frac{1}{2}\left[\left(\left(2 \mathrm{~N}_{z} \mathrm{H}_{x} \pm \mathrm{H}_{x}\right) \frac{1}{2} s^{-}+\left(2 \mathrm{~N}_{z} \mathrm{H}_{y} \pm \mathrm{H}_{y}\right) \frac{1}{2} c^{-}\right)\right. \\
& \left.-\left(\left(-\mathrm{H}_{x} \mp 2 \mathrm{H}_{x} \mathrm{~N}_{z}\right) \frac{1}{2} s^{-}+\left(\mathrm{H}_{y} \pm 2 \mathrm{H}_{y} \mathrm{~N}_{z}\right)\left(-\frac{1}{2} c^{-}\right)\right)\right] ;
\end{aligned}
$$

condition (b)

$$
\begin{aligned}
\frac{1}{2}\left[\left(\left(2 \mathrm{~N}_{z} \mathrm{H}_{x} \mp \mathrm{H}_{x}\right)\left(-\frac{1}{2} s^{+}\right)+\left(2 \mathrm{~N}_{z} \mathrm{H}_{y} \mp \mathrm{H}_{y}\right) \frac{1}{2} c^{+}\right)\right. \\
\left.-\left(\left(-\mathrm{H}_{x} \pm 2 \mathrm{H}_{x} \mathrm{~N}_{z}\right) \frac{1}{2} s^{+}+\left(\mathrm{H}_{y} \mp 2 \mathrm{H}_{y} \mathrm{~N}_{z}\right) \frac{1}{2} c^{+}\right)\right] \\
-\frac{1}{2}\left[\left(\left(2 \mathrm{~N}_{z} \mathrm{H}_{x} \pm \mathrm{H}_{x}\right)\left(-\frac{1}{2} s^{-}\right)+\left(2 \mathrm{~N}_{z} \mathrm{H}_{y} \pm \mathrm{H}_{y}\right) \frac{1}{2} c^{-}\right)\right. \\
\left.-\left(\left(-\mathrm{H}_{x} \mp 2 \mathrm{H}_{x} \mathrm{~N}_{z}\right) \frac{1}{2} s^{-}+\left(\mathrm{H}_{y} \pm 2 \mathrm{H}_{y} \mathrm{~N}_{z}\right) \frac{1}{2} c^{-}\right)\right] .
\end{aligned}
$$


Table 1

Observable magnetization obtained by the pulse sequence of Fig. 1a (first scan) under different gradient and phase conditions

\begin{tabular}{lrcc}
\hline Gradient & $\phi_{1}$ & $\sigma\left(t_{1}, 0\right)$ & Peak $^{\mathrm{a}}$ \\
\hline$G_{\mathrm{selN}}=-\frac{\gamma_{\mathrm{H}}}{\gamma_{\mathrm{N}}} G_{\mathrm{selH}}$ & $y$ & $-\frac{1}{2}\left[\left(2 \mathrm{~N}_{z} \mathrm{H}_{x}+\mathrm{H}_{x}\right) \sin \left(\omega_{\mathrm{N}} t_{1}-\pi J t_{1}\right)+\left(2 \mathrm{~N}_{z} \mathrm{H}_{y}+\mathrm{H}_{y}\right) \cos \left(\omega_{\mathrm{N}} t_{1}-\pi J t_{1}\right)\right]$ & $\mathrm{aT}$ \\
& $-y$ & $+\frac{1}{2}\left[\left(2 \mathrm{~N}_{z} \mathrm{H}_{x}+\mathrm{H}_{x}\right) \sin \left(\omega_{\mathrm{N}} t_{1}+\pi J t_{1}\right)+\left(2 \mathrm{~N}_{z} \mathrm{H}_{y}+\mathrm{H}_{y}\right) \cos \left(\omega_{\mathrm{N}} t_{1}+\pi J t_{1}\right)\right]$ & $\mathrm{ec} 2$ \\
$G_{\mathrm{SelN}}=\frac{\gamma_{\mathrm{H}}}{\gamma_{\mathrm{N}}} G_{\mathrm{SelH}}$ & $y$ & $+\frac{1}{2}\left[\left(\mathrm{H}_{x}-2 \mathrm{~N}_{z} \mathrm{H}_{x}\right) \sin \left(\omega_{\mathrm{N}} t_{1}+\pi J t_{1}\right)+\left(2 \mathrm{~N}_{z} \mathrm{H}_{y}-\mathrm{H}_{y}\right) \cos \left(\omega_{\mathrm{N}} t_{1}+\pi J t_{1}\right)\right]$ & $\mathrm{T}$ \\
& $-y$ & $-\frac{1}{2}\left[\left(\mathrm{H}_{x}-2 \mathrm{~N}_{z} \mathrm{H}_{x}\right) \sin \left(\omega_{\mathrm{N}} t_{1}-\pi J t_{1}\right)+\left(2 \mathrm{~N}_{z} \mathrm{H}_{y}-\mathrm{H}_{y}\right) \cos \left(\omega_{\mathrm{N}} t_{1}-\pi J t_{1}\right)\right]$ & $\mathrm{ec} 1$
\end{tabular}

${ }^{\mathrm{a}}$ For the nomenclature definition see Fig. $1 \mathrm{~b}$.

Expressions (6) and (7) can be rearranged to yield the four magnetization states that are obtained just before the detection time, by combining the polarity of the two gradients $G_{\text {selN }}$ and $G_{\text {selH }}$ and the phase $\phi_{1}$ (Table 1). As can be observed by inspection of the relative signs of the two components of each term, and the relative signs of the $\Omega_{\mathrm{N}}$ and $\pi J$ evolution during $t_{1}$, the single scan of the pulse sequence of Fig. 1a contains selectively one of the four different combinations. Magnetization terms obtained with $\phi_{1}=y$ correspond to the trosy (with cross-peaks located at $\left.\left(\pi J+\omega_{\mathrm{N}}, \pi J-\omega_{\mathrm{H}}\right)\right)$ or the anti-trosy component (cross-peaks located at $\left.\left(\pi J-\omega_{\mathrm{N}}, \pi J+\omega_{\mathrm{H}}\right)\right)$, whereas those obtained with $\phi_{1}=-y$ are the two e-cosy components (located at $\left(\pi J+\omega_{\mathrm{N}}, \pi J+\omega_{\mathrm{H}}\right)$ and $\left.\left(\pi J-\omega_{\mathrm{N}}, \pi J-\omega_{\mathrm{H}}\right)\right)$. The product operator analysis shows that no phase cycle is needed to obtain each one of the four components of the $\mathrm{H}-\mathrm{N}$ system. For practical reasons, mainly to obtain a minimal residual water signal, it is advisable to perform an inversion of phase $\Psi_{1}$ with the consequent inversion of the detector phase, reducing to two scans the complete phase cycle of the experiment.

In the four experiments, the magnetization is obtained as a mixture of the real (cosine modulated) and imaginary (sine modulated) components. In all cases, a second fid is obtained by changing the polarity of $G_{\text {selN }}$ and increasing $180^{\circ}$ phases $\phi_{1}$ and $\Psi_{2}$. Data manipulation in echo, anti-echo manner and Fourier transformation, yields a 2D spectrum in pure absorption mode in the two dimensions.

As a first application example, we have applied this approach to the measurement of RDCs of a ternary complex between the chicken liver bile acid binding protein and two molecules of chenodeoxycholate. A selected region that superimposes the four different spectra obtained by combination of gradient polarity and $\phi_{1}$ phase is shown in Fig. $1 b$.

\subsection{Compensation for relaxation-induced artifacts}

It was already shown that TROSY pulse sequences are prone to relaxation-induced artifacts at the positions of the other components of the $\mathrm{H}-\mathrm{N}$ doublet [22,25]. A slight modification was proposed to the original pulse sequence, in order to compensate for the relaxation-induced artifacts by coherently generating exactly the same artifacts but with opposite phase [25]. We have tested for the presence of these artifacts when applying the sequence of Fig. 1a varying the delay duration and pulse phases originally proposed [25]. Figure 2a shows some examples of the occurrence of artifacts in the spectra containing the $\mathrm{T}$ and aT peaks. Although they don't represent more than 5\% of the total intensity of the main peak, their presence can be deleterious when high accuracy in the peak position is desirable for the determination of the coupling constants. Mainly two parameters can be adjusted in order to reduce the artifact intensity: the delay $\tau_{2}$ and the phase $\Psi_{2}$ (see Fig. 1a). We have performed a series of experiments recording the $\mathrm{T}$ peak (using condition $\mathrm{b}$ for gradients polarity and $\phi_{1}=y$ ), and varying systematically the duration of $\tau_{2}$ and $\Psi_{2}$. Results are presented in Fig. 2b, where the graphics show the 


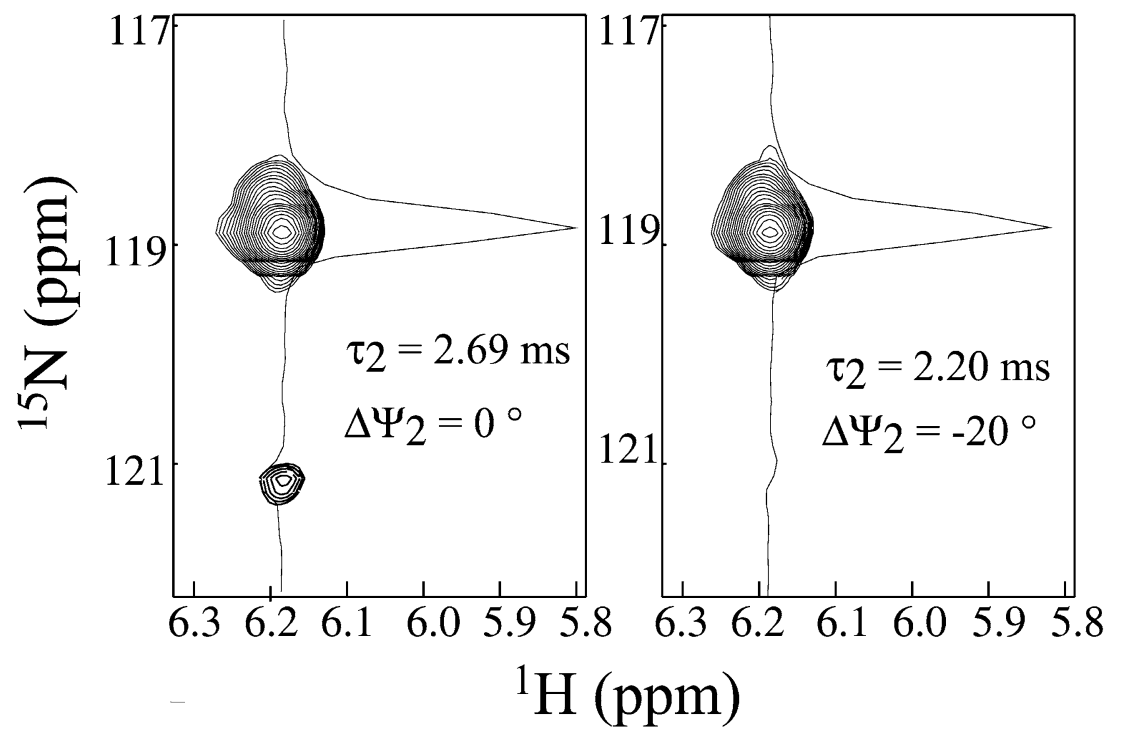

(a)

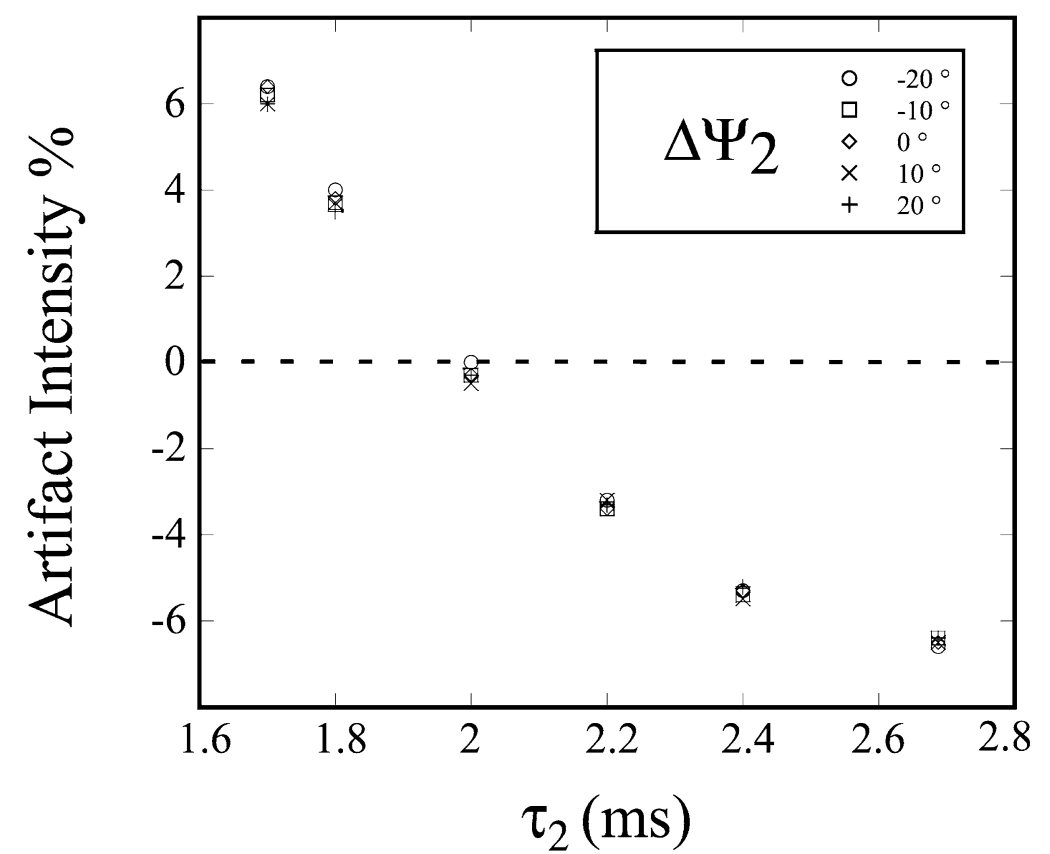

(b)

Fig. 2. (a) Selected region of the ${ }^{15} \mathrm{~N} /{ }^{13} \mathrm{C}$-labeled cl-BABP TROSY spectrum showing the presence of artifacts at the position of ec2 peak. (b) Artifacts are strongly reduced when setting $\tau_{2}$ to a shorter value ( $2.20 \mathrm{~ms}$ in this specific case) and when changing the $\Psi_{2}$ phase ( -20 degree in this specific case). One-dimensional extractions parallel to the $F_{1}$ axis through the ${ }^{15} \mathrm{~N}-{ }^{1} \mathrm{H}$ selected peak are overlayed on the 2D spectrum. (c) Plot reporting the average intensity ratio of artifacts to TROSY peak intensity, expressed as percentage value, as a function of $\tau_{2}$ delay at distinct $\Psi_{2}$ phase correction values. The two parameters are chosen for the minimum artifact intensity. 
relative intensity of the artifact with respect to the $\mathrm{T}$ peak as a function of both parameters. In our hands, changing the phase $\Psi_{2}$ has little effect. By using a slightly shorter delay for $\tau_{2}$ we were able to reduce significantly the artifact intensity. The optimum duration of this delay to minimize artifact intensity has to be obtained empirically, because it depends on the relaxation properties of the nuclei under study, and on the spectrometer field. Similar results were obtained for all four experiments, indicating that a single correction of $\tau_{2}$ is sufficient to ensure a clean selectivity.

\subsection{Data acquisition and analysis}

The four different experiments giving rise to $\mathrm{T}, \mathrm{aT}$, ec1 and ec2 peak types were encoded into a single pseudo-3D experiment, the TEC experiment, in which the four $2 \mathrm{D}$ spectra are acquired in a fully interleaved manner to reduce differences in peak position arising from spectrometer and/or sample instabilities. All four spectra show an excellent water suppression, due to the use of gradients to select the ${ }^{15} \mathrm{~N}$ coherence pathway.

After Fourier Transformation, both the aT and ec 2 spectra need to be reversed in the $F_{1}$ dimension. This is a direct consequence of the fact that magnetization yielding $\mathrm{T}$ or aT peaks (or ec1 and ec2 peaks) shows after $t_{1}$ evolution an opposite sign for the sine component (see equation (2)). For an accurate estimation of the coupling constants from relative peak displacements in the four spectra, care must be taken when processing the reversed spectra, specially if software like NMRView [20] is used. It uses the central point for the reference value, and, if the number of processed points is even, it assumes the $1+n / 2$ point as the reference point, where $\mathrm{n}$ is the number of points. This introduces a systematic deviation equal to the point separation in $\mathrm{Hz}$ to the observed peak differences in the $N$-dimension. By shifting by one unit the reference point of the reversed spectrum, or by using $2^{m}+1$ points in the transformation, this problem can be simply solved.

Coupling constants between $H$ and $N$ were measured by using the sequence of Fig. 1a, in a sample of labelled chicken liver bile acid binding protein complexed with chenodeoxycholate. Measurements were obtained both in $\mathrm{H}_{2} \mathrm{O}$ and in a partially aligned sample using Pf1 as alignment medium [21]. In principle, coupling constants can be obtained in many different ways, depending on the pairs of peaks used to estimate the shifts. We have measured twice the ${ }^{1} J_{\mathrm{HN}}$ in the N-dimension using differences between $\mathrm{T}-\mathrm{aT}$ and ec1-ec2 peak types. The same was repeated for the $\mathrm{H}$-dimension.

When comparing the values obtained for the sample in $\mathrm{H}_{2} \mathrm{O}$, it can be noticed that the average value for the ${ }^{1} \mathrm{~J}_{\mathrm{HN}}$ scalar couplings measured in the $\mathrm{N}$-dimension is higher $(93.4 \pm 0.8 \mathrm{~Hz})$ than the one obtained from the $\mathrm{H}$-dimension $(91.9 \pm 1.5 \mathrm{~Hz})$. This deviation was already observed [16], and, at least partially, it was explained by the unsymmetrical distortion of peaks in the H-dimension. The distortion arises from cross-correlation effects that yields different intensities for the two components of the $\mathrm{HN}-\mathrm{H} \alpha$ doublet, altering the position of the peak maximum. This effect is less pronounced for Gly residues, as there are two $\mathrm{HN}-\mathrm{H} \alpha$ couplings giving rise to a doublet of doublets. In fact, the average values measured in the two dimensions for Gly residues are closer one to the other $(93.2 \pm 0.8 \mathrm{~Hz}$ and $93.9 \pm 0.6 \mathrm{~Hz}$ for the $\mathrm{H}$ and $\mathrm{N}$-dimensions, respectively), than those obtained for all residues.

There are, however, other less systematic contributions that in general makes less accurate the measurement of coupling constants in the $\mathrm{H}$-dimension. They include phase imperfections and passive $\mathrm{H}-\mathrm{H}$ couplings that make the peak broader. This is specially evident for peaks in aligned samples, because in addition to the $\mathrm{HN}-\mathrm{H} \alpha$ scalar coupling, there are contributions of other $\mathrm{H}-\mathrm{H}$ dipolar couplings to the ${ }^{1} \mathrm{H}$ linewidth. 

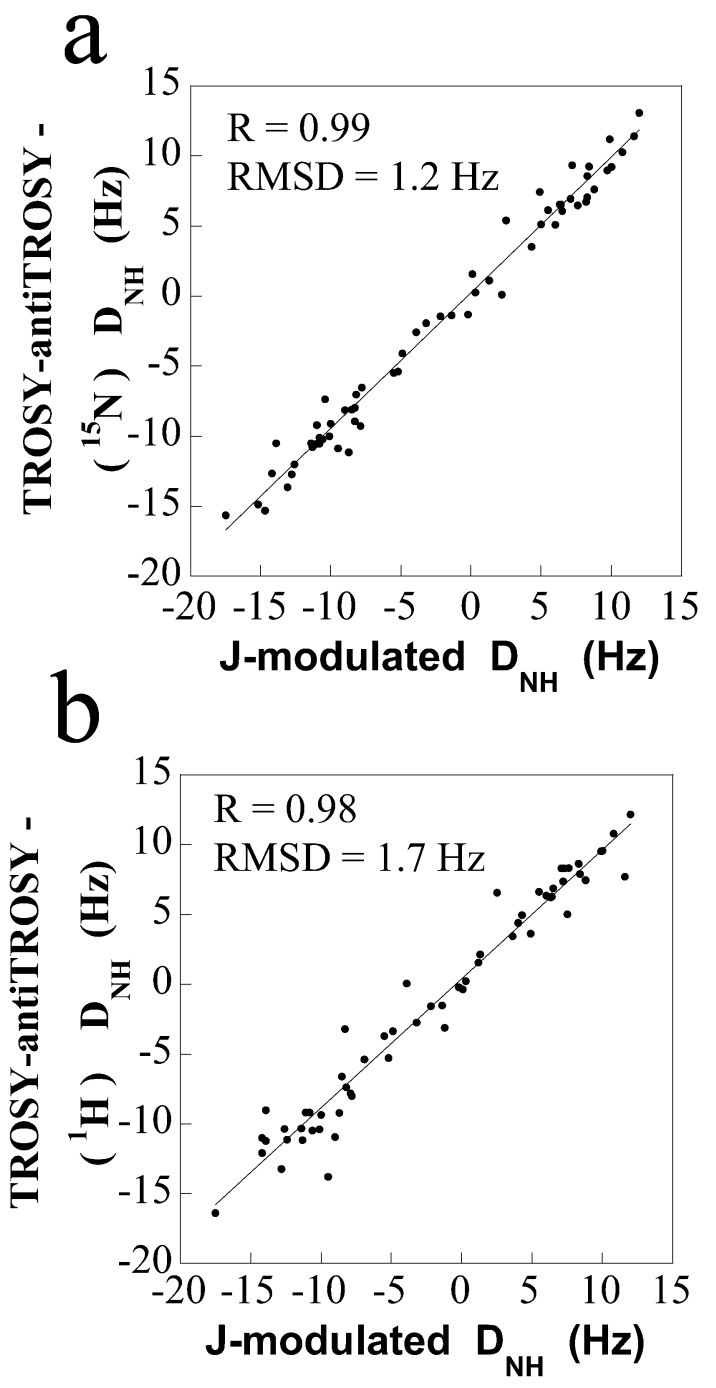

Fig. 3. Correlation of RDCs measured using the TROSY-antiTROSY experiment (a) along the ${ }^{15} \mathrm{~N}$ dimension and (b) along the ${ }^{1} \mathrm{H}$ dimension with RDCs obtained from $J$-modulation method. The correlation factor $R$ for the linear regression and the RMSD between the corresponding RDCs are also reported.

\subsection{RDC measurement using the T-aT and ec1-ec2 crosspeaks}

RDC values were calculated as the difference between the ${ }^{1} J_{\mathrm{HN}}$ observed in $\mathrm{H}_{2} \mathrm{O}$ and in the aligned sample. In order to estimate the accuracy of the measurements using the different spectra and dimensions, we have used a $J$-modulation experiment [23] to measure independently the RDC values of the protein. Figure 3 shows the correlation between the values observed for the $\mathrm{N}$ - and $\mathrm{H}$-dimensions using the T-aT peaks and those observed in the intrinsically more accurate data obtained with $J$-modulation. Table 2 summarises the observed RMSD between the different measurements. The values obtained in the $\mathrm{N}$-dimension turn out to be more accurate than those in the H-dimension. Moreover, by taking the average value of the two differences ( $\mathrm{T}-\mathrm{aT}$ and ec1-ec2) the RMSD is reduced with respect to the 
Table 2

Observed RMSD between different measurements using the experiments depicted in Fig. 1a compared to the $J$-modulation experiment [23]

\begin{tabular}{lcccccccc}
\hline & \multicolumn{3}{c}{ N-dimension } & & \multicolumn{3}{c}{ H-dimension } \\
\cline { 2 - 3 } & T-aT & ec1-ec2 & Average & & T-aT & ec1-c2 & Average \\
\hline RMSD $[\mathrm{Hz}]$ & 1.2 & 1.2 & 1.0 & & 1.7 & 1.7 & 1.6 \\
\hline
\end{tabular}

$J$-modulation measurement for the $\mathrm{N}$-dimension (a $F$-test gave a $p=0.03$ that the reduction occurred by chance), but not for the H-dimension.

The low RMSD obtained for the best measurement using the T-aT, ec1-ec2 average values in the $\mathrm{N}$-dimension indicates an acceptable accuracy of this method, even when compared with more time consuming measurements, like $J$-modulation. In the latter, not less than 16 to 20 different $2 \mathrm{D}$ experiments are necessary to evaluate the modulation induced by the ${ }^{1} J_{\mathrm{HN}}$ coupling constant [23]. The RMSD value of $1.0 \mathrm{~Hz}$ represents an error of $3 \%$ of the total range of couplings observed for the sample here considered. In general, it is enough to estimate the RDCs with a precision of less than $10 \%$, because this is the inherent limit imposed by the inaccuracy of the peptide models that are used to translate the RDC information into structural features [4]. In terms of resolution, since both approaches are based on 2D experiments, they both yield a similar number of sufficiently resolved peaks that can be used for the measurement.

\subsection{Comparison with the IPAP experiment}

The IPAP scheme explores the possibility of obtaining in two different spectra the two components of the HN doublet, as the ${ }^{1} J_{\mathrm{HN}}$ is active only during $t_{1}$ evolution [8]. With respect to the experiments here presented, the IPAP spectra yields peaks with integral larger by a factor of 2, as the total magnetization is split into two instead of four. Sensitivity enhanced versions of the IPAP experiments are now also available [16]. On the other hand, as Table 2 suggests, the TEC experiment here presented allows for a number of independent measurements of the coupling constants, shifting the peaks in opposite directions. This gives more than one chance of obtaining sufficient peak resolution in at least one of the several combination of experiments. For example, Fig. 4 shows that peaks corresponding to N60 and L78 could not be resolved in the aT spectrum, but their position can be accurately determined in the ec1-ec2 spectra. Examples like this are more frequent in the aligned sample, because the coupling constants do not present an uniform value, giving rise to superposition of peaks that were not superimposed in the normal ${ }^{1} \mathrm{H}-{ }^{15} \mathrm{~N}$ HSQC spectrum and vice versa.

Using delay compensation for artifact reductions, in our hands the peak separation into different subspectra is cleaner in the case of the TEC experiment. The IPAP experiment suffers from the presence of difference artifacts that are difficult to eliminate, and that depend on differential relaxation behaviour and different coupling constants of individual HN signals.

Finally, the TEC experiment can be used for a wider range of molecular weights. Larger proteins show broader aT peaks, making the measurement less accurate using the IPAP experiment. The contribution of the aT linewidth is present in the IPAP experiment giving rise to a broader up-field component. This contribution is less important when analyzing the ec1-ec2 peaks, that show an intermediate line-width between the $\mathrm{T}$ and aT components.

To illustrate this point we have recorded the four subspectra at $700 \mathrm{MHz}$ for a $30 \mathrm{kDa}$ protein, the Azotobacter vinelandii Rhodanese [18], and the results are shown in Fig. 5. For comparison, also the IPAP experiment was performed on the same sample, and utilizing the same experimental time. Inspection of 


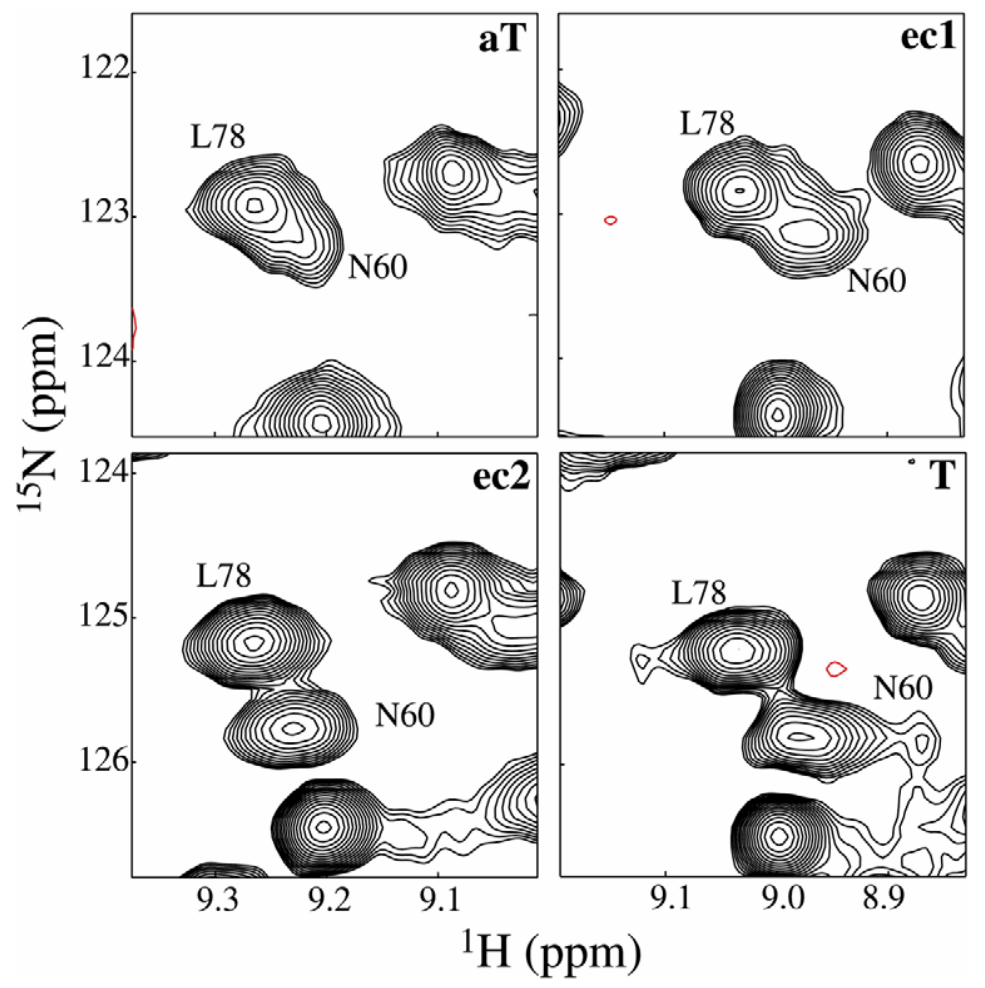

Fig. 4. An expanded region of the four TROSY-type spectra showing that peak resolution can benefit from two independent measurements ( $\mathrm{T}-\mathrm{aT}$ and ec1-ec2). In the specific case, when using only T-aT experiment, cross-peaks of L68 and N70 overlap to some extent in one of the two spectra, whereas a clean peak separation is obtained in the ec1, ec 2 experiments.

Fig. 5 allows to conclude that the best results, in terms of resolution, are obtained when using the $\mathrm{T}$ and the two e-cosy peaks. In this way, two independent measurements of the ${ }^{1} J_{\mathrm{HN}}$ can be obtained, and in this particular case, a larger number of couplings could be estimated by using this strategy, compared to the IPAP experiment. As can be observed, the high field component of the HN doublet in the IPAP spectrum is broad, thereby heavily impairing the resolution of a large number of peaks, particularly in crowded regions of the spectrum.

\section{Conclusion}

We have shown that slight modifications of the gradient selected trosy experiments can be used to extract any of the four subspectra that compose the $\mathrm{HN}-\mathrm{N}$ doublet. Artifact reduction can be implemented by simply tuning the duration of a time delay. The use of gradient selection allows an excellent water suppression. This approach is perfectly suited for medium size proteins (of molecular weight up to $20 \mathrm{kDa}$ ). For larger proteins, as peaks get broader (particularly the aT component), the definition of peak positions becomes less accurate. However, a separate inspection of TROSY (T), the narrowest component, and E-COSY (ec1, ec2), the components with intermediate line-widths, allows in most cases the extension of the methodology to larger proteins, compared to all other previously discussed methods. 


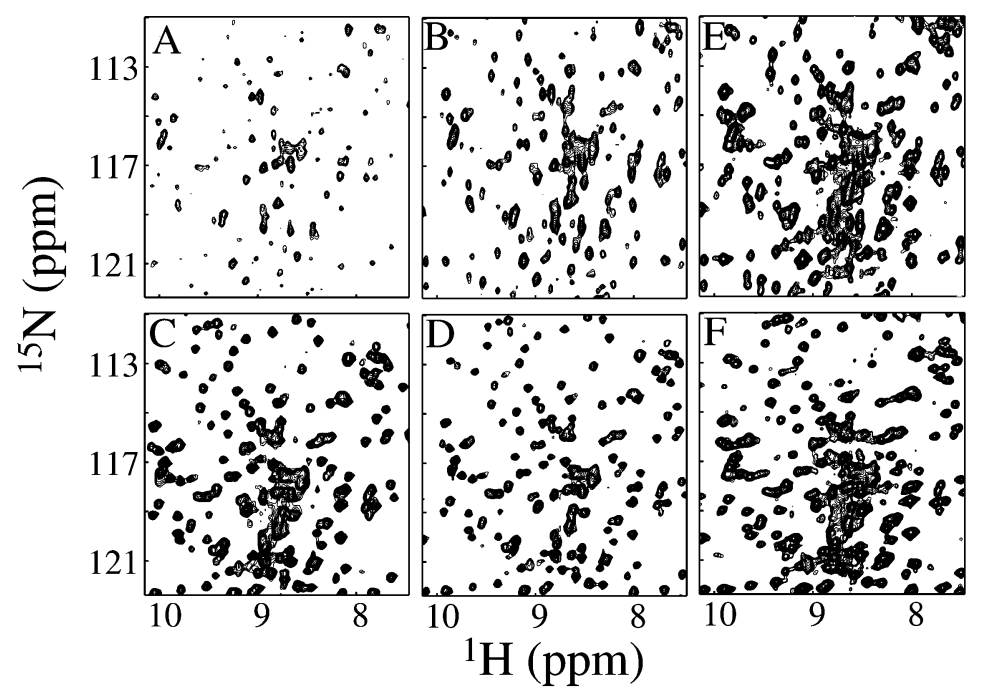

Fig. 5. Comparative performance of the aT, ec1, ec2, T experiments (panels A-D, respectively) and the IPAP experiment (panels E-F) using a $30 \mathrm{kDa}$ perdeuterated protein at $700 \mathrm{MHz}$, Rhodanese from Azotobacter vinelandii. The T subspectrum (panel D) shows the best resolution and is used as pivot to calculate the coupling constant, using as second peaks both the ec1 (panel B) and ec2 (panel C) peaks, that show a better resolution than the up-field component of the IPAP experiment (panel E). In this way, two independent measurements of the couplings, with enhanced resolution, can be obtained. The six spectra are plotted showing the same relative level with respect to noise.

\section{Acknowledgment}

We are grateful to Laura Ragona, Lucia Zetta and Henriette Molinari and Sonia Melino for kindly providing the protein samples.

\section{References}

[1] A. Bax and A. Grishaev, Weak alignment NMR: a hawk-eyed view of biomolecular structure, Curr. Opin. Struct. Biol. 15 (2005), 563-570.

[2] A. Bax, G. Kontaxis and N. Tjandra, Dipolar couplings in macromolecular structure determination, Methods Enzymol. 339 (2001), 127-174.

[3] F. Rodriguez-Castaneda, P. Haberz, A. Leonov and C. Griesinger, Paramagnetic tagging of diamagnetic proteins for solution NMR, Magn. Reson. Chem. 44 Spec. No (2006), S10-16.

[4] J.H. Prestegard, K.L. Mayer, H. Valafar and G.C. Benison, Determination of protein backbone structures from residual dipolar couplings, Methods Enzymol. 394 (2005), 175-209.

[5] J.A. Lukin, G. Kontaxis, V. Simplaceanu, Y. Yuan, A. Bax and C. Ho, Quaternary structure of hemoglobin in solution, Proc. Natl. Acad. Sci. USA 100 (2003), 517-520.

[6] N. Tjandra and A. Bax, Measurement of dipolar contributions to ${ }^{1} \mathrm{~J}_{\mathrm{CH}}$ splittings from magnetic-field dependence of $\mathbf{J}$ modulation in two-dimensional NMR spectra, J. Magn. Reson. 124 (1997), 512-515.

[7] N. Tjandra, S. Grzesiek and A. Bax, Magnetic field dependence of nitrogen-proton J splittings in ${ }^{15} \mathrm{~N}$-enriched human ubiquitin resulting from relaxation interference and residual dipolar coupling, J. Am. Chem. Soc. 118 (1996), 6264-6272.

[8] M. Ottiger, F. Delaglio and A. Bax, Measurement of $J$ and dipolar couplings from simplified two-dimensional NMR spectra, J. Magn. Reson. 131 (1998), 367-372.

[9] P. Andersson, J. Weigelt and G. Otting, Spin-state selection filters fort he measurement of heteronuclear one-bond coupling constants, J. Biomol. NMR 12 (1998), 435-441.

[10] B. Brutscher, Accurate measurement of small spin-spin couplings in partially aligned molecules using a novel J-mismatch compensated spin-state-selection filter, J. Magn. Reson. 151 (2001), 332-338. 
[11] K. Pervushin, R. Riek, G. Wider and K. Wüthrich, Attenuated $T_{2}$ relaxation by mutual cancellation of dipole-dipole coupling and chemical shift anisotropy indicates an avenue to NMR structures of very large biological macromolecules in solution, Proc. Natl. Acad. Sci. USA 94 (1997), 12366-12371.

[12] L. Kay, P. Keifer and T. Saarinen, Pure absorption gradient enhanced heteronuclear single quantum correlation spectroscopy with improved sensitivity, J. Am. Chem. Soc. 114 (1992) 10663-10665.

[13] M. Czisch and R. Boelens, Sensitivity enhancement in the TROSY experiment, J. Magn. Reson. 134 (1998), 158-160.

[14] J. Weigelt, Single scan, sensitivity- and gradient-enhanced TROSY for multidimensional NMR experiments, J. Am. Chem. Soc. 120 (1998), 10778-10779.

[15] K.V. Pervushin, G. Wider and K. Wüthrich, Single transition-to-single transition polarization transfer (ST2-PT) in [15N,1H]-TROSY, J. Biomol. NMR 12 (1998), 345-348.

[16] K. Ding and A.M. Gronenborn, Sensitivity-enhanced 2D IPAP, TROSY-anti-TROSY, and E.COSY experiments: alternatives for measuring dipolar ${ }^{15} \mathrm{~N}-{ }^{1} \mathrm{H}^{\mathrm{N}}$ couplings, J. Magn. Reson. 163 (2003), 208-214.

[17] L. Ragona, M. Catalano, M. Luppi, D. Cicero, T. Eliseo, J. Foote, F. Fogolari, L. Zetta and H. Molinari, NMR dynamic studies suggest that allosteric activation regulates ligand binding in chicken liver bile acid binding protein, J. Biol. Chem. 281 (2006), 9697-9709.

[18] M. Gallo, S. Melino, R. Melis, M. Paci and D.O. Cicero, Backbone NMR assignment of the 29.6 kDa Rhodanese protein from Azotobacter vinelandii, J. Biomol. NMR (2006), in press.

[19] F. Delaglio, S. Grzesiek, G.W. Vuister, G. Zhu, J. Pfeifer and A. Bax, NMRPipe: a multidimensional spectral processing system based on UNIX pipes, J. Biomol. NMR 6 (1995) 277-293.

[20] B.A. Johnson and R.A. Blevins, NMRView: A computer program for the visualization and analysis of NMR data, J. Biomol. NMR 4 (1994), 603-661.

[21] M.R. Hansen, L. Mueller and A. Pardi, Tunable alignment of macromolecules by filamentous phage yields dipolar coupling interactions, Nat. Struct. Biol. 5 (1998), 1065-1074.

[22] M. Rance, L.J. Patrick and A.G. Palmer III, Sensitivity improvement of transverse relaxation-optimized spectroscopy, J. Magn. Reson. 136 (1999), 92-101.

[23] R.L. McFeeters, C.A. Fowler, V.V. Gaponenko and R.A. Byrd, Efficient and precise measurement of $\mathrm{H}^{\alpha}-\mathrm{C}^{\alpha}, \mathrm{C}^{\alpha}-\mathrm{C}^{\prime}$, $\mathrm{C}^{\alpha}-\mathrm{C}^{\beta}$ and $\mathrm{H}^{\mathrm{N}}-\mathrm{N}$ residual dipolar couplings from $2 \mathrm{D} \mathrm{HN}-\mathrm{N}$ correlation spectra, J. Biomol. NMR 31 (2005), 35-47.

[24] O.W. Sørensen, G.W. Eich, M.H. Levitt, G. Bodenhausen and R.R. Ernst, Product operator formalism for the description of NMR pulse experiments, Progress in NMR Spectroscopy 16 (1983), 163-192.

[25] T. Schulte-Herbrüggen and O.W. Sørensen, Clean TROSY: compensation for relaxation-induced artifacts, J. Magn. Reson. 144 (2000), 123-128. 


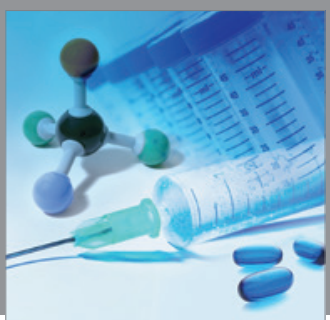

International Journal of

Medicinal Chemistry

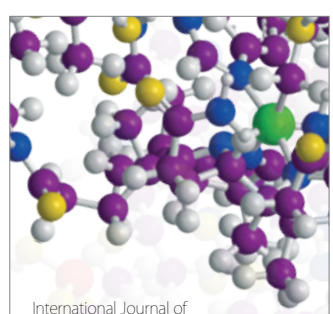

Carbohydrate Chemistry

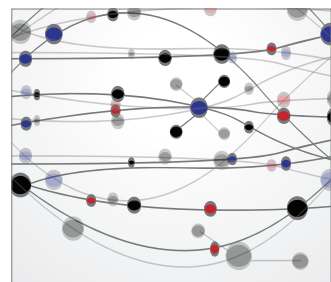

The Scientific World Journal
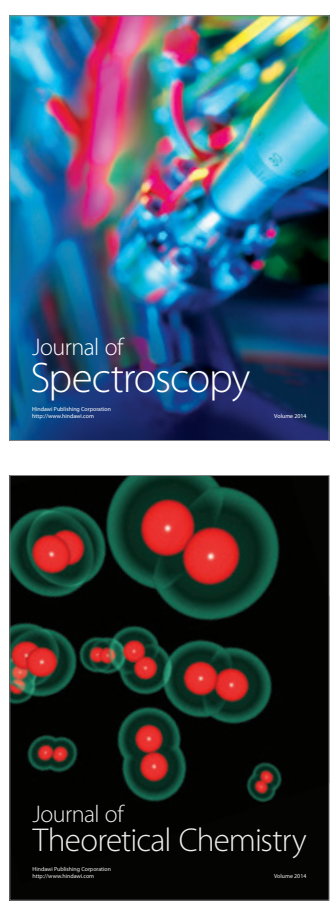
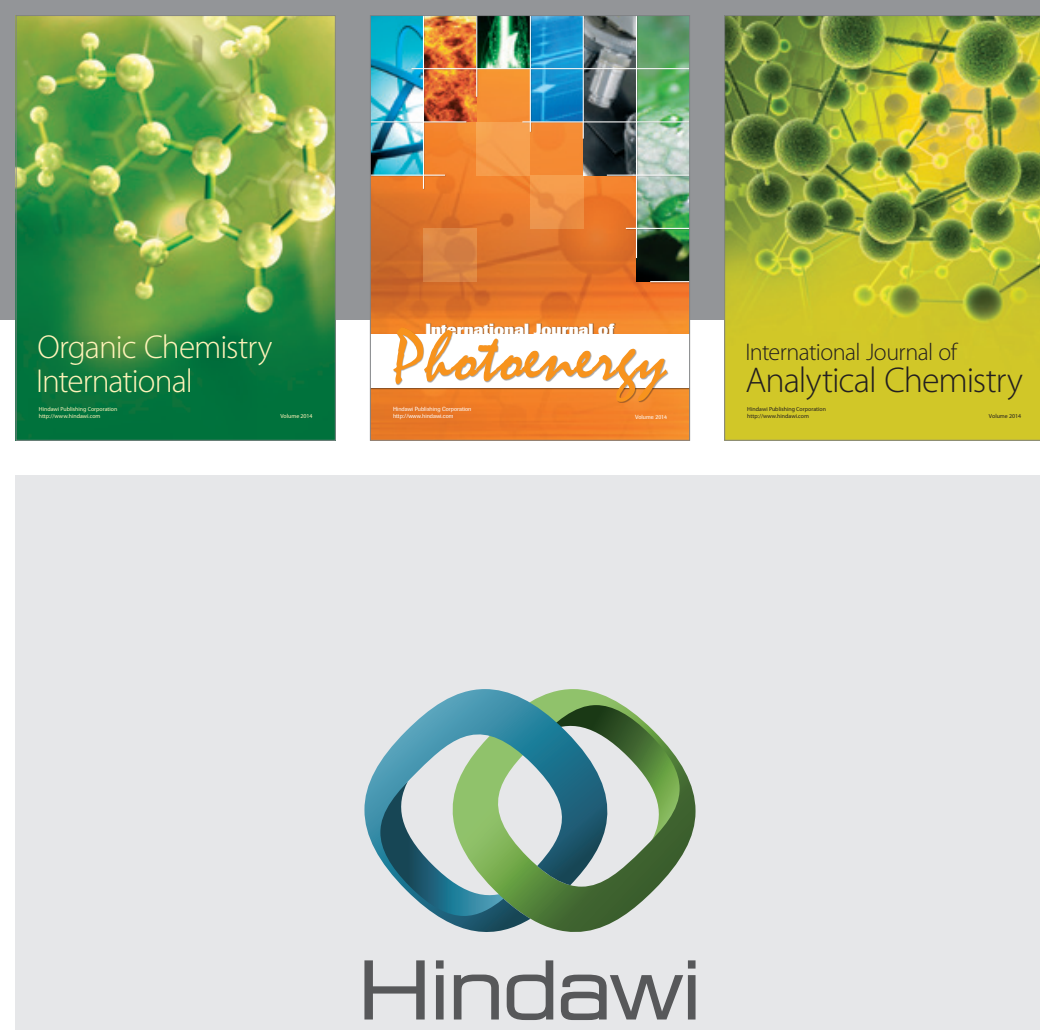

Submit your manuscripts at

http://www.hindawi.com
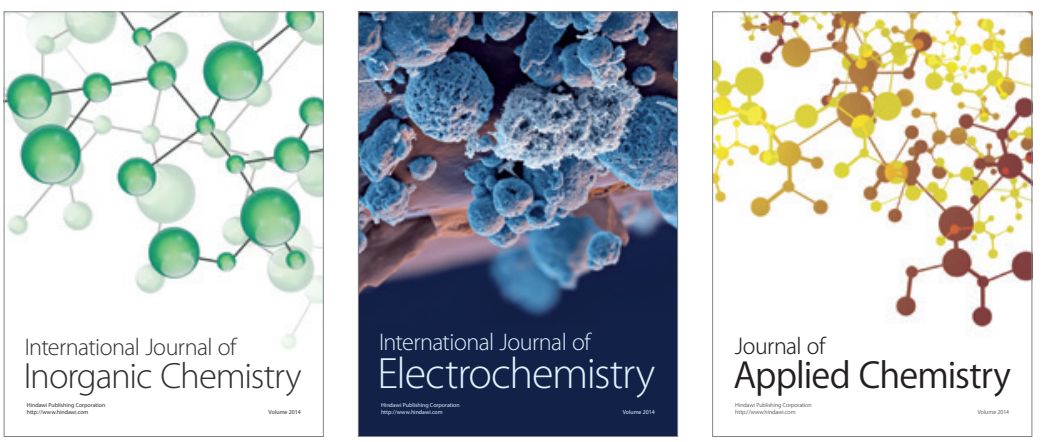

Journal of

Applied Chemistry
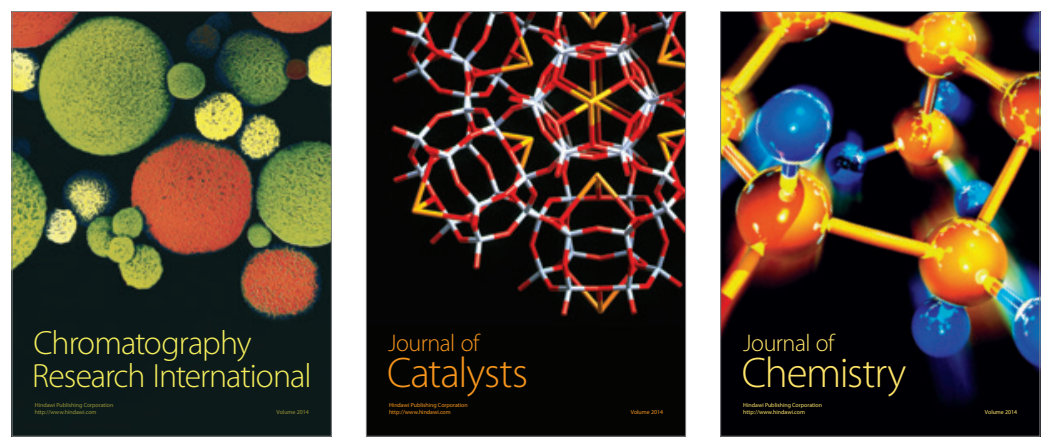
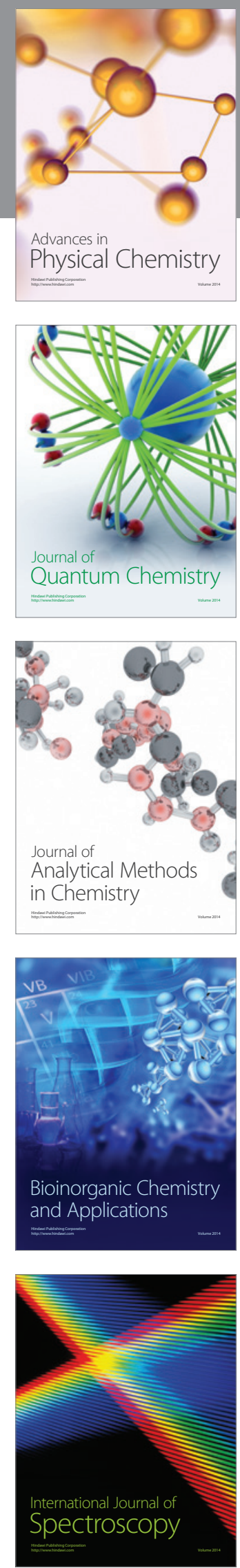